\section{DEVICE FOR FIGURE-OF-EIGHT SUTURES}

\section{Alfred Hull Clark, M.D., Buffalo}

Figure of eight silkworm-gut sutures, used to reinforce the fascial catgut stitch, may be tied over a wire frame longer than the skin incision and as wide as the distance between the skin holes of any stitch. This wire frame allows inspection of the line of skin sutures and the treatment of any irregularity in its healing, prevents the cutting of the skin by the silkworm-gut, obviates any pressure on the skin incision, if it be held with skin clamps, and gives an even pull on $t$ he fascia without pressure on the $\mathrm{skin}$ other than the slight and well-distributed pressure of the frame.

The frame is made of No. 35 brass wire, $\mathrm{n}$ ickeled, is $2 \mathrm{t} / 2$ inches wide and varies in length to suit the skin incision. Those used by Dr. Earl P. Lothrop, with
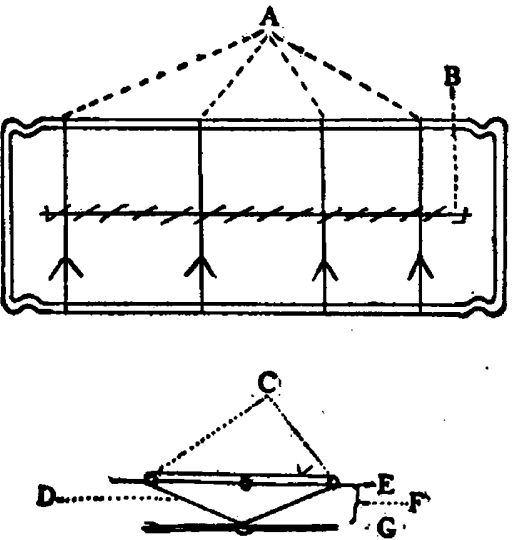

Above, diagram of frame, one-third size. $A$, silkworm-gut; $B$, skin incision. Below, diagram of skin, fascia and frame in cross section, one-third size. $C$, frame; $D$, figureof-eight silkworm-gut; $E$, skin; $F$, fat; $G$, fascia.

whom I work, vary from $3 \frac{1}{4}$ to $7 \frac{1}{2}$ inches in length. The accompanying diagram illustrates the frame and its use. The idea was adapted from Rovsing's glass plate.

153 Delaware Avenue.

\section{ISOLATION OF BACILLUS TUBERCULOSIS FROM SPUTUM BY THE METHOD OF PETROFF*}

\section{O. W. H. Mitchell, M.D., And R. R. Simmons, A. M.,} Syricuse, N. Y.

Recently Petroff ${ }^{1}$ described a method for the isolation of the tubercle bacillus from sputum and feces. It gave highly satisfactory results, particularly when isolation from sputum was attempted. With sixty-nine specimens of known tuberculous sputums he isolated the organism in every instance and, with a little different technic, he was successful in isolating Bacillus tuberculosis from feces nineteen times out of thirty-two attempts.

The method will not be described in detail as it will be best for the reader, if the method is to be employed, to refer to the original article. By using an alkaline solution to which $B$. tuberculosis is especially resistant and employing gentian violet, which inhibits the growth of many organisms, Petroff succeeded in perfecting a method for tubercle bacillus isolation which is simple, economical and trustworthy, especially when the isolation is to be made from sputum. In brief the method is as follows:

Digest the sputum in sodium hydroxid solution in the incubator at $37.5 \mathrm{C}$. for from twenty to thirty minutes. Neutralize to litmus with normal hydrochloric acid and centrifugalize. Spread several loops of the sediment on beef-infusion-glycerin-egg medium to which has been added gentian violet in the strength of $1: 10,000$. Petroff used fresh sputum, while we have used sputum from one to four days old.

Attempts have been made in this laboratory to isolate the tubercle bacillus on the medium without gentian violet. This method was used twenty-three times, but because of con-

* From the Bacteriological Laboratory, College of Medicine, Syracuse University.

1. Petroff: Jour. Exper. Med., 1915, xxi, No. 1, p. 38 tamination only once was the tubercle bacillus grown in pure culture. Several times growth was noted by making smears but, excepting one culture, contamination was marked. Petroff was more successful and without gentian violet he isolated $B$. tuberculosis five times out of twenty trials.

Gentian violet was added and with thirty-five specimens our results were as follows: isolation and good growth, twenty-eight; contamination, six; no growth, one. Growth was observed in the different cultures from the ninth to the fifteenth day. Later development proved that the observation of early growth was correct. As the failures occurred at the beginning of the work we are inclined to consider them due to faulty technic rather than to defects in the method. All specimens of sputum employed in this work were shown by smears to be positive for $B$. tuberculosis.

Such a method which makes animal inoculation unnecessary and can be so simply performed commends itself for wide application, especially in the teaching laboratory.

\section{A NEW POCKET COLORIMETER *}

\section{Theodore Kuttner, M.D., New York}

In making analyses of blood and other physiologic fluids, it is often desirable to employ small quantities of the material to be tested, provided accurate microchemical methods with suitable instruments are available. Several advantages would result, namely, first, the ability to use less reagent for the test; second, the saving of time and labor; third, the probability of more often obtaining such material, and finally the use of less costly apparatus.

The object of this communication is to describe an instrument for use in quantitatively estimating small amounts of a constituent in solution by its color reaction. Before proceeding with the description, I wish to state that the same calibrated and color tube employed in the Sahli-Gower hemoglobinometer has been adopted for this instrument. While the Sahli allows both tubes to be viewed at full length, the new instrument shows only a small part of the tubes when making color comparison. This facilitates the color reading and is less fatiguing to the eye. An additional feature is a prism $(A)$ to be described, which has the effect of causing the contents of the tubes to approach each other more closely, thereby a c complish ing greater accuracy in comparing the colors.

The instrument (now being made by $E$. Leitz, New York) consists of a closed upright box, the dimensions of which are about $2.5 \mathrm{~cm}$. in width by $8.5 \mathrm{~cm}$. in height, being therefore smaller in length and width than the Sahli instrument. At the top are two openings, one for the color tube, $D$, and the other for the calibrated tube, $E$. Near the lower front part of the instrument is a window provided with a small prism, $A$ (Helmholtz double p l a te s), above mentioned,

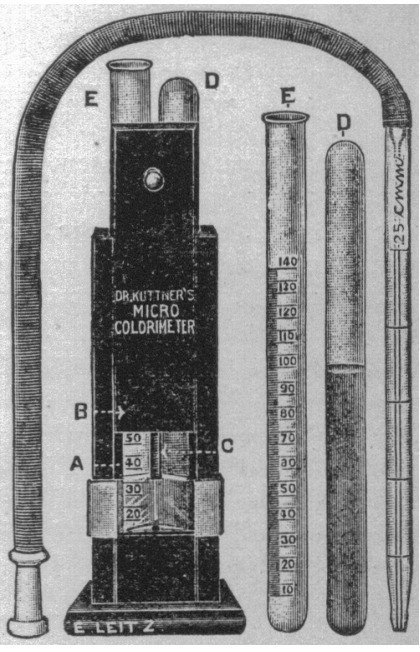

A pocket colorimeter. serving the purpose of having the colors of both tubes appear close together, forming a continuous color band. The prism is easily removed for cleansing and can be quickly readjusted. A sliding door, $B$, which can be raised and lowered, protects the prism from dust and injury. The color standard and calibrated tube are separted from each other by a partition, $C$, which prevents light from being reflected from one tube to the other.

* From the Pathological Laboratory, Department of Physiological Chemistry, Mt. Sinai Hospital. 